\title{
Rheological Study of Polymer--Colloid Complexes of Aluminoxane Particles with Weakly Charged Polyelectrolytes in Semidilute Aqueous Solutions
}

\author{
Stanislav Sergeevich Radchenko (Corresponding author) \\ Department of Analytical, Physical Chemistry and Physical-Chemistry of Polymers \\ Volgograd State Technical University \\ 28 Lenina av., 400055, Russian Federation
}

Tel: 7-108-844-224-8068 E-mail: radchenko@vstu.ru

Novakov Ivan Aleksandrovich

Department of Analytical, Physical Chemistry and Physical-Chemistry of Polymers

Volgograd State Technical University

28 Lenina av., 400055, Russian Federation

E-mail: rector@vstu.ru

Philipp Stanislavovich Radchenko

Department of Analytical, Physical Chemistry and Physical-Chemistry of Polymers

Volgograd State Technical University

28 Lenina av., 400055, Russian Federation

Tel: 7-108-844-224-8068Ｅ-mail: radchenko@vstu.ru

Aleksandr Sergeevich Ozerin

Department of Analytical, Physical Chemistry and Physical-Chemistry of Polymers

Volgograd State Technical University

28 Lenina av., 400055, Russian Federation

E-mail: asozerin@mail.ru

Ol'ga Alekseevna Manoylina

Department of Analytical, Physical Chemistry and Physical-Chemistry of Polymers

Volgograd State Technical University

28 Lenina av., 400055, Russian Federation

E-mail: manoylinaolya@yandex.ru

Received: December 29, 2011 Accepted: January 10, 2012 Published: Apri1 1, 2012

doi:10.5539/jmsr.v1n2p119

URL: http://dx.doi.org/10.5539/jmsr.v1n2p119

\begin{abstract}
Viscoelastic properties of hydrogels based on polymer-colloid complexes of aluminoxane particles with acrylamide-acrylic acid copolymers were studied. Rheometric measurements were performed with an MFR 2000 Fourier microrheometer (GBS, Australia) under the regime of oscillating loads with the use of the dynamic mechanical analysis. It is shown that, with copolymer concentrations above the crossover point, the phase angle
\end{abstract}


decreases abruptly; that is, the elastic characteristics of the gels decline. The maximum elastic properties of the gels for all copolymers are attained at the molar ratio of reagents $Z \approx 1$. The effect of strain rate on relaxation processes in the gels was investigated. It is found that, at low strain rates, irreversible deformations (flow) can take place, and with an increase in frequency, the strength of physical networks formed with participation of aluminoxane particles tends to increase.

Keywords: Copolymers, Aluminoxane particles, Gelation, Rheological properties

\section{Introduction}

Many publications are devoted to the study of gelation in water--polymer systems (hydrogels) (Gidley, 1989; Goddard, 1993; Erdodi, Janecska \& Jvan, 1999; Tighe, 2000; Picot \& Ross-Murphy, 2000; Rogovina, Vasil'ev \& Braudo, 2008; Pavljuchenko \& Ivanchev, 2009; Wu et al., 2009; Novoskol'ceva, et al., 2009; Kurmaz \& Ozhiganov, 2010). Hybrid hydrogels containing inorganic particles in aqueous solutions of polymers in the bound-dispersed state have been studied to a smaller extent, although this new type of polymer networks makes it possible to obtain materials which structure and properties are untypical for common network polymers (Makarov, Andreeva \& Tretinnik, 2001; Averchenko, Papisov \& Matvienko, 1993; Kurt, et al., 2005; Kirillina, et al., 2009; Vinogradov, Agaphonov \& Vinogradov, 2010). There are some characteristic features that distinguish formation of hybrid hydrogels from development of purely polymeric gels. This is due to the fact that the dispersions of colloid particles, which are lyophobic sols, are thermodynamically unstable. Only some of them, which contain on the surface of particles acidic or basic groups capable of ionization with a change in the $\mathrm{pH}$ of aqueous solutions, may be rather stable against aggregation in a certain $\mathrm{pH}$ range due to the presence of the double electric layer. Examples of these dispersed systems are sols of aluminum polyhydroxochloride containing aluminoxane particles (APs) with characteristic sizes of 4-6 nm (Zakharchenko, et al., 2006; Ozerin, et al., 2009) and forming polycomplexes with a number of water-soluble polymers (Novakov, Radchenko \& Papisov, 2005 \& 2007; Novakov, et al., 2006 \& 2011; Radchenko, 2011). The rheological behavior of these systems depends on concentration of a polymer, its properties, presence of intra- and intermolecular bonds, amount of the crosslinking agent, etc. The necessary condition for chemically crosslinked gels to be formed is the achievement of a certain minimum concentration of the polymer, at which the hydrodynamic volumes of macromolecules begin to overlap and the "infinite" cluster appears in the reaction system (Gidley, 1989). Evidently, the same situation holds true for physically crosslinked gels; that is, the onset of structure formation corresponds to the moment, when macromolecules or their polycomplexes turn out to be bound via topological entanglements within the whole volume of solution. In the case of hybrid hydrogels, two mechanisms of gel formation develop during formation of uniform spatial networks (Averchenko, Papisov \& Matvienko, 1993). The first mechanism is realized as a result of the aggregation of poorly soluble fragments of macromolecules bound to the surface of sol particles. A necessary condition for this effect to occur is that the length of macromolecular chains should significantly exceed the sizes of sol particles so that several particles could reside on one macromolecule. The gels formed according to this mechanism are nonuniform. They are characterized by low strength and are poorly recovered after mechanical breakdown. In terms of the second mechanism of gel formation, one particle is bound with more than one polymer macromolecule and, at the same time, one macromolecule is bound with more than two particles. This mechanism may be implemented if sizes of macromolecule coils and sol particles are commensurable, concentrations of macromolecules and particles are close, and distances between macromolecules and particles are comparable with their sizes. The gels formed according to this mechanism are uniform and semi-transparent and feature high mechanical strength. In terms of these notions, the rheological study of polymer-colloid complexes is of great importance. These complexes belong to dispersed systems with specific viscous-flow characteristics, and their known applications as waterproofing gel compositions and binding agents (Novakov, et al., 2008; Radchenko, et al., 2009; Samrej, et al., 2011) presuppose their participation in the processes of filtration and interaction with porous media. The viscoelastic properties of dilute and semi-dilute solutions are the key factors affecting these processes.

\section{Experimental}

\subsection{Starting Materials}

The dispersion of APs was prepared from aluminum alloy in the form of aqueous solutions as described in (Novakov et al., 1997). The content of $\mathrm{Al}^{3+}$ was $12.6 \mathrm{wt} \%$, and the $\mathrm{Cl}$ : $\mathrm{Al}$ atomic ratio was 0.46 . Ultra dry sodium chloride ( $99.99 \%$, Alfa Aesar, USA) was used. All experiments were performed using bidistilled water.

\subsection{Synthesis of Polymers}

Aqueous solutions of copolymers (CPs) of acrylamide with acrylic acid containing $0.1,0.2,0.4$, and $0.6 \mathrm{~mol} \%$ acrylic acid units (accordingly, CP-0.1, CP-0.2, CP-0.4, and CP-0.6) were prepared and characterized as described in (Novakov, et al., 2011). The characteristics of the copolymers are listed in Table 1. 


\subsection{Preparation of Water--Salt Solutions of Copolymer-AP Sol Mixtures with the Invariable Concentration of Copolymer but Different Concentrations of AP Sol}

Seven volumetric $25-\mathrm{cm}^{3}$ flasks were charged each with aqueous solutions of CP-0.1 (5.83 g, $3.0 \mathrm{wt} \%$ ), aqueous solutions of $\mathrm{NaCl}\left(0.5 \mathrm{~mol} / 1,0.5 \mathrm{~cm}^{3}\right)$, and distilled water $(10 \mathrm{ml})$. The mixtures were stirred, and the aqueous solution of the AP sol $\left(1.21 \mathrm{~mol} / 1\right.$ based on $\left.\mathrm{Al}^{3+}\right)$ was added $\left(0.2,0.4,1.0,1.6,2.4,5\right.$, and $10 \mathrm{~cm}^{3}$, respectively). Distilled water was added to the contents in the flasks to bring the volume to the filling mark. The prepared solutions were allowed to stand for a day to attain equilibrium. For CP-0.2-CP-0.6 copolymers, the solutions of mixtures were prepared in a similar manner except the concentrations of the AP sol were $2.42 \times 10^{-3}, 4.84 \times 10^{-3}$, $7.26 \times 10^{-3} \mathrm{~mol} / 1$ based on $\mathrm{Al}^{3+}$ for $\mathrm{CP}-0.2, \mathrm{CP}-0.4, \mathrm{CP}-0.6$, respectively.

\subsection{Preparation of Water--Salt Solutions of Copolymer Mixtures with the AP Sol of Different Concentrations but} Invariable Concentration Ratio between $\mathrm{Al}^{3+}$ and AA Units

Five volumetric $25-\mathrm{cm}^{3}$ flasks were charged each with $3.0 \mathrm{wt} \%$ aqueous solution of CP-0.1 (2.5, 3.3, 4.2, 5.0, $5.8 \mathrm{~cm}^{3}$, respectively), aqueous $\mathrm{NaCl}$ solution $\left(0.5 \mathrm{~cm}^{3}, 0.5 \mathrm{~mol} / \mathrm{l}\right)$, and distilled water $(10 \mathrm{ml})$. The mixtures were stirred, and the aqueous solution of AP sol with a concentration of $1.0 \mathrm{~mol} / 1$ based on $\mathrm{Al}^{3+}$ was added (1.0, $1.3,1.6,2.0$, and $2.3 \mathrm{~cm}^{3}$, respectively). Distilled water was added to the contents in the flask to bring the volume to the filling mark. The prepared solutions were allowed to stand for a day to attain equilibrium. The solutions of mixtures for CP- 0.2 - CP- 0.6 copolymers were prepared in a similar manner except the concentrations of AP sol were $2.00 \times 10^{-3}, 4.00 \times 10^{-3}, 6.00 \times 10^{-3} \mathrm{~mol} / 1$ based on $\mathrm{Al}^{3+}$ for CP-0.2, CP-04, CP-0.6, respectively.

2.5 Investigations of Viscoelastic Behavior of Water-Salt Solutions of the Mixtures of Copolymers with AP Dispersion

Viscoelastic behavior was studied with the aid of an MFR 2000 Micro Fourier Rheometer (GBC, Australia). The measurements were performed in the frequency range from 0 to $199 \mathrm{~Hz}$ with a step of $1 \mathrm{~Hz}$ and at a temperature $25^{\circ} \mathrm{C}$. All variable parameters were entered in the authorized MFR 2100 ver. $1.1 \mathrm{~b} 4$ program.

The main operating unit of the rheometer was composed of two 7- and 1.25-mm-diam disks which were arranged horizontally one over another (the smaller disk was located above). A certain amount of the gel sufficient for covering the whole surface was placed on the larger disk. The small disk descended until contact with the gel surface and then began to execute forced oscillations. The large disk was fastened motionlessly and was connected with a sensor recording the response of the gel - the shear stress to desired oscillations. Then, through Fourier transformations of shear stress and shear strain signals, the frequency dependences of dynamic storage modulus, dynamic viscosity, and phase angle were calculated.

The values of phase angle between the shear stress and the shear strain for viscoelastic liquids may vary from $\pi / 2$ (viscous medium) to 0 (elastic medium). For systems containing polymers and colloid particles, both types of response to the applied shear stress are possible. A sharp change in the value of phase angle between the shear stress and the shear strain was taken as a starting point of gelation, at which response of a medium to mechanical impact becomes elastic.

\section{Results and Discussion}

The study of formation of polymer--colloid complexes of positively charged aluminoxane particles with oppositely charged linear polyelectrolytes in dilute aqueous solutions revealed that soluble polymer-colloid complexes are formed with macromolecules containing a small amount of ionogenic groups (not above 0.8 mol \%) (Novakov, et al., 2011). Formation of complexes is accompanied by the compaction of macromolecular coils, while aluminoxane particles become uniformly distributed over polyelectrolyte macromolecules. In this case, the dispersion system on the whole represents the true solution without any phase separation in a wide range of molar ratios of reagents.

Upon the addition of nanosized aluminoxane particles to the solution of polyelectrolyte, which concentration was close or equal to that of the crossover point, gel formation, that is, formation of the uniform network of intermacromolecular bonds, was observed. The process of gel formation was investigated by the method of dynamic mechanical analysis (DMA).

Figure 1 depicts the dependences of phase angle for water-salt mixtures of copolymers containing different amounts of acrylic acid (AA) units and APs on composition Z. These plots were measured at an oscillation frequency of $20 \mathrm{~Hz}$. The concentration of the copolymers was invariable and equal to $0.7 \mathrm{~g} / \mathrm{dl}$. This value was slightly higher than that of the crossover point. 
The definition of mixture composition $\mathrm{Z}$ was given in (Novakov et al., 2011). This is the ratio between the number of positively charged groups $\left[\mathrm{Al}^{+}\right]$located on the particle surface and the number of AA units in solution $\mathrm{Z}=\left[\mathrm{Al}^{+}\right] /\left[\mathrm{COO}^{-}\right]$.

As it is seen from Figure 1, addition of the water-salt solution of APs leads to a sharp decrease in the phase angle. This is indicative of the formation of spatial crosslinks between copolymer macromolecules. In this case, nanosized aluminoxane particles play the role of crosslinking agents. The larger the amount of AA units in the copolymer, the sharper the decline in dependence of phase angle on $Z$, and the stronger the elastic properties. It should be noted that for all polymers elastic properties are achieved at $Z \approx 1$.

Figure 2 demonstrates the dependences of phase angle for water-salt mixtures of different copolymers with APs at invariable $\mathrm{Z}$ on the concentration of the mixture at the same frequency of oscillations $(20 \mathrm{~Hz})$.

The above-described data show that, up to the crossover concentration, the phase angle changes insignificantly and remains practically invariable. At concentrations above the crossover point, the phase angle begins to decrease rapidly, thereby indicating that the elastic reaction of the medium prevails over the viscous component.

It may be suggested that, in the region up to the crossover concentration, PCCs are formed by macromolecules and particles so that for each copolymer macromolecule there is a certain number of aluminoxane particles which are bound only with one copolymer macromolecule. At the concentrations close to the crossover concentration or above, an aluminoxane particle is bound with several copolymer macromolecules and forms intermolecular crosslinks. The as-prepared gels are uniform and semi-transparent and are distinguished with high mechanical strength. Thus, the mechanism of gel formation presumably implies that more than one polymer macromolecule may be bound with every particle, whereas more than two particles may be bound with every macromolecule.

It is also interesting to analyze the dependence of viscoelastic properties of the gels on the shear strain---the frequency of oscillations. The optimal composition Z of PCCs for all copolymers is equal to 1 . Therefore, we investigated the dependences of viscoelastic properties of compositions with different concentrations of the copolymer (from 0.1 to $1 \mathrm{~g} / \mathrm{dl}$ ) but with the invariable composition, namely, at $Z=\underline{1}$.

The dependences of the dynamic storage modulus (Figure 3) and the mechanical loss tangent (Figure 4) on the frequency of oscillations demonstrate that, with an increase in the rate of shearing, the elasticity of gels on the whole grows monotonically. At the same time, there is the specific feature in the behavior of the gels, namely, there is a break on the frequency dependence of the storage modulus. At a frequency of $70 \mathrm{~Hz}$, the elasticity of the gels begins to increase sharply. Such a behavior is especially well pronounced for the gel with a lower copolymer concentration. For the gel with a higher copolymer concentration, the maximum of mechanical losses is clearly seen at a frequency $80 \mathrm{~Hz}$. For the gel with a smaller copolymer concentration, the position of maximum of mechanical losses is evidently beyond the limits of the operating frequency range of the measuring instrument $(100 \mathrm{~Hz})$.

Such a viscoelastic behavior provides experimental evidence that relaxation processes occur in the gels under study. It is probable that, at low strain rates, irreversible deformations, that is, flow, at which particles can migrate relatively freely along the chains of macromolecules, are still possible. With an increase in the frequency of oscillations, the process of particle migration decelerates (the particles mostly oscillate relative to the equilibrium position), and the strength of the physical network formed with participation of aluminoxane particles increases.

\section{References}

Averchenko, I. A., Papisov, I. M., \& Matvienko, V. M. (1993). Gelation of aqueous sols of polysilicic acid with some polymers. Polymer Science, Ser A, 35, 1986-1990.

Erdodi, G., Janecska, A., \& Jvan, B. (1999). Hovel Intelligent Amphiphilic Co networks. Polymer Networks Group Review Series, Vol. 2, Wiley, New-York.

Gidley, M. J. (1989). Molecular mechanisms underlying amylose aggregation and gelation. Macromolecules, 22, 351-358. http://dx.doi.org/10.1021/ma00191a064

Goddard, E. D. (1993). Interaction of Surfactants with Polymer and Proteins. Roca Raton, CRC.

Kirillina, U. O., Bakeeva, I. V., Bulichev, N. A., \& Zubov, V. P. (2009). Organic-inorganic hybrid hydrogels based on linear poly(N-vinylpyrrolidone) and products of hydrolytic polycondensation of tetramethoxysilane. Polymer Science, Ser B., 51, 135-142. http://dx.doi.org/10.1134/S1560090409030105 
Kurmaz, S. V., \& Ozhiganov, V. V. (2010). Polymer networks prepared via the crosslinking free-radical polymerization of dimethacrylate in the presence of branched polymethacrylate and its fractions. Polymer Science, Ser B, 52, 369-380. http://dx.doi.org/10.1134/S1560090410070018

Kurt, V. D., Bruno, V. M., Wonter, L., \& Filip, E. Du. (2005). Prez Introduction of silica into thermo-responsive poly (N-isopropyl acrylamide) hydrogels: A novel approach to improve response rates. Polymer, 46, 9851-9862. http://dx.doi.org/10.1016/j.polymer.2005.08.032

Makarov, S. A., Andreeva, I. A., \& Tretinnik, V. U. (2001). Rheological properties of polymer-containing aqueous aerosil dispersions. Colloid Journal, 63, 731-737. http://dx.doi.org/10.1023/A:1013223920360

Novakov, I. A., Radchenko, Ph. S., \& Papisov, I. M. (2005). The properties of aqueous solutions of polymer-colloid complexes of polyacrylamide with poly (aluminum hydroxychloride). Polymer Science, Ser A, 47, 57-60.

Novakov, I. A., Radchenko, Ph. S., \& Papisov, I. M. (2007). A study of the composition of polyacrylamide-polyaluminum chloride polymer-colloid complexes. Polymer Science, Ser B, 49, 111-113. http://dx.doi.org/10.1134/S1560090407050016

Novakov, I. A., Radchenko, Ph. S., Ozerin, A. S., Rybakova, E. V., \& Radchenko, S. S. (2011). Formation of polymer-colloid complexes of aluminoxane particles with poly(acrylic acid) and its copolymers with acrylamide. Colloid Polym. Sci., 289, 1197-1203. http://dx.doi.org/10.1007/s00396-011-2446-4

Novakov, I. A., Radchenko, S. S., Bykadorov, N. U., Kargin, U. N., Mochov, V. Ph., Zhochova, O. K., Parchomenko A. I., \& Otchenashev P. I. (1997). RF Patent 2083495 JPC E21B 33136.

Novakov I. A., Radchenko S. S., Pastukhov A. S., \& Radchenko Ph. S. (2006). Properties of aqueous solutions of polymer-colloid complexes of polyethylenimine with aluminum hydroxychloride. Russian Journal of Applied Chemistry, 80, 1906-1909

Novakov, I. A., Radchenko, S. S., Radchenko, Ph. S., Ozerin, A. S., Karaulov, A. V. (2008). Polyacrylamide-aluminum pentahydroxochloride-urea formulations as waterproofing agents for oil pool. Russian Journal of Applied Chemistry, 81, 1465-1468. http://dx.doi.org/10.1134/S1070427208080272

Novoskol'ceva O. A., Rogacheva V. B., Zezin A. B., Joostan J., \& Brackman J. (2009). Formation and transformations of polyelectrolyte gel-ampholyte dendrimer-surfactant ternary complexes. Polymer Science, Ser A, 51, 598-605. http://dx.doi.org/10.1134/S0965545X09060030

Ozerin, A. S., Radchenko, Ph. S., Timofeeva, G. I., \& Novakov, I. A. (2009). A study of structural and molecular weight characteristics of poly(aluminum hydroxychloride) nanoparticles by small-angle X-ray scattering and sedimentation analysis. Nanotechnologies in Russia, 4, 93-101. http://dx.doi.org/10.1134/S1995078009010108

Pavljuchenko, V. N., \& Ivanchev, S. S. (2009). Composite polymer hydrogels. Polymer Science, Ser. A., 51, 743-760. http://dx.doi.org/10.1134/S0965545X09070013

Picot, D. R., \& Ross-Murphy, S. B. (2000). Polymer Gels and Networks. Ed. by Osada Y, Khokhlov A. Dekker, New-York.

Radchenko, S. S., Novakov, I. A., Radchenko, Ph. S., Le Van Cong, Ozerin, A. S., \& Zel'tser, P. S. J. (2011). Interaction of aluminoxane particles with weakly charged cationic polyelectrolyte. J. Appl. Polym. Sci., 121: 475-482. http://dx.doi.org/10.1002/app.33597

Radchenko, S. S., Novakov, I. A., Radchenko, Ph. S., Ozerin, A. S., Zel’tser, P. S., \& Yakubovski, S. U. (2009). RF Patent 2348792 JPC E21B 33136.

Rogovina, L. Z., Vasil'ev, V. G., \& Braudo, E. E. (2008). Definition of the concept of polymer gel. Polymer Science, Ser C, 50, 85-92. http://dx.doi.org/10.1134/S1811238208010050

Samrej, I. A., Kidalov, N. A., Radchenko, Ph. S., Radchenko, S. S., Osipova, N. A., \& Zacutaev, V. A. (2011). The polymer-colloidal complex as a binding material for casting molds and cores manufacturing. Technika Mashinostroen, 2, 31-34.

Tighe, B., \& Silicone, Hydrogels. (2000). The Rebirth of Continuons Wear Contact Lenses. Ed. by Sweeney D. Oxford, P.J.

Vinogradov, V. V., Agaphonov, A. V., \& Vinogradov, A. V. (2010). Sol-gel synthesis of nanostructured materials based on aluminum oxide with preset texture properties. Protection of Metals and Physical Chemistry of Surfaces, 46, 582-586. http://dx.doi.org/10.1134/S207020511005014X 
Wu, H., Zaccone, A., Tsountsoura, A., Lattuada, M., \& Morbidelli, M. (2009). High Shear-Induced Gelation of Charge-Stabilized Colloids in a Microchannel without Adding Electrolytes. Langmuir, 25, 4715-4723. http://dx.doi.org/10.1021/la803789s

Zakharchenko, S. O., Litmanovich, O. A., Radchenko, Ph. S., Pastukhov, A. S., Zezin, A. B., Novakov, I. A., \& Kabanov, V. A. (2006). Photon correlation spectroscopic study of the aggregative stability of colloidal particles of aluminum pentahydroxide chloride. Colloid Journal, $68, \quad 425-429$. http://dx.doi.org/10.1134/S1061933X06040053

Table 1. The main characteristics of acrylamide-acrylic acid copolymers

\begin{tabular}{|l|c|c|c|}
\hline Copolymer & $\begin{array}{c}\text { Content of acrylic acid units in } \\
\text { copolymer, mol \% }\end{array}$ & Molecular mass & Crossover concentration, g/dl \\
\hline CP-0.1 & 0.1 & $5.7 \times 10^{5}$ & 0.59 \\
\hline CP-0.2 & 0.2 & $5.5 \times 10^{5}$ & 0.56 \\
\hline CP-0.4 & 0.4 & $5.6 \times 10^{5}$ & 0.62 \\
\hline CP-0.6 & 0.6 & $6.1 \times 10^{5}$ & 0.57 \\
\hline
\end{tabular}

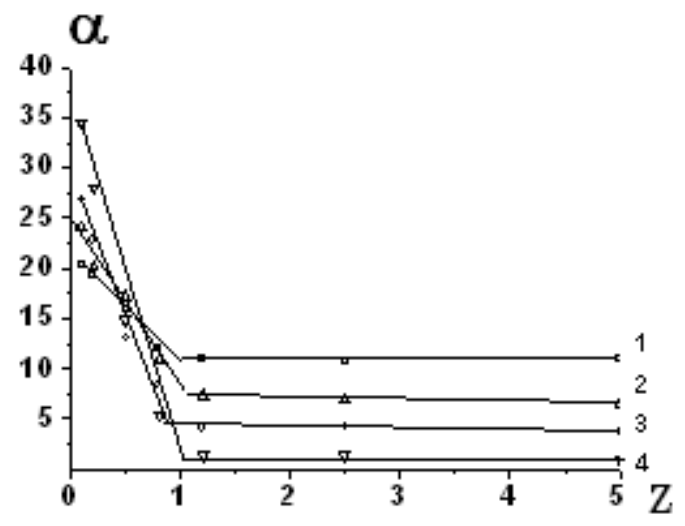

Figure 1. Dependence of phase angle for water-salt solutions of the copolymer mixtures with APs on mixture composition Z. The contents of AA units in the copolymer are (1) 0.1 , (2) 0.2 , (3) 0.4 , and (4) $0.6 \mathrm{~mol} \%$. The concentration of $\mathrm{NaCl}$ is $0.01 \mathrm{~mol} / 1$. The concentration of the copolymers is $0.7 \mathrm{~g} / \mathrm{dl}$. The frequency of oscillations is $20 \mathrm{~Hz}$

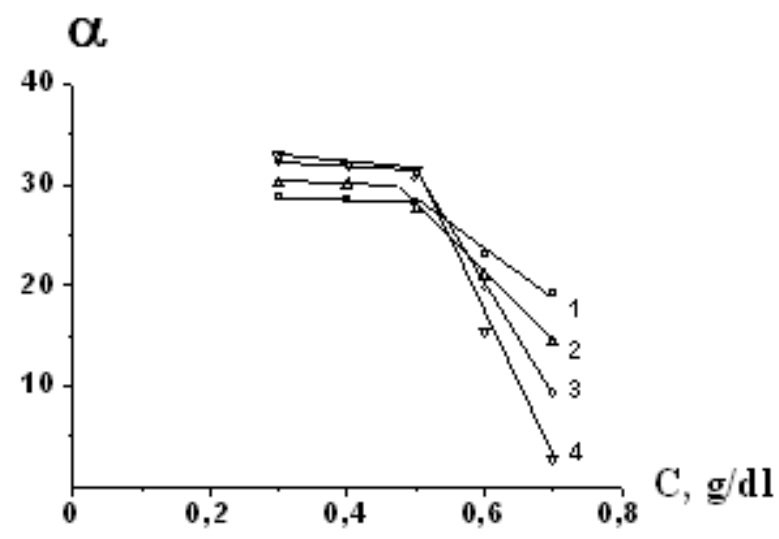

Figure 2. Dependence of phase angle for water--salt solutions of copolymer mixtures with the aqueous dispersion of APs at constant $\mathrm{Z}=1$ on mixture concentration. Contents of AA units in the copolymer (mol \%) are: (1) 0.1, (2) 0.2, (3) 0.4 , and (4) $0 / 6$. The concentration of $\mathrm{NaCl}$ is $0.01 \mathrm{~mol} / \mathrm{l}$. The oscillation frequency is 20 


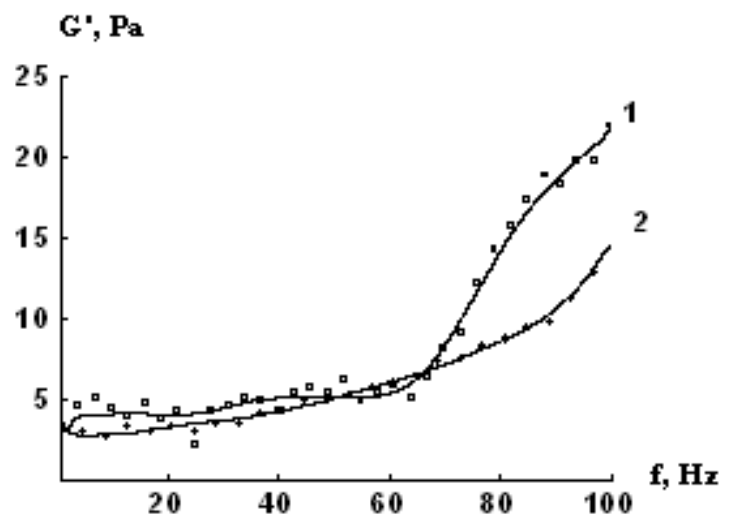

Figure 3. Frequency dependence of dynamic storage modulus of PCCs $(Z=1)$ with a change in the concentration of the complexed copolymer: (1) 0.1 and (2) $1.0 \mathrm{~g} / \mathrm{dl}$

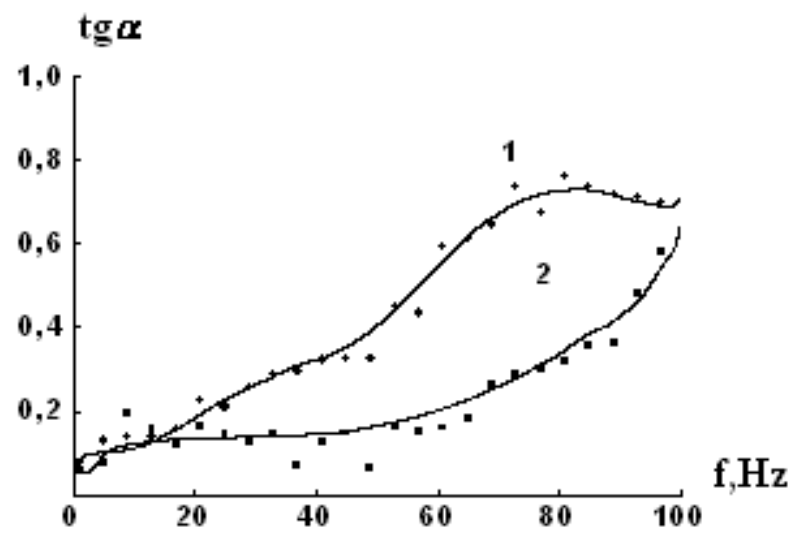

Figure 4. Frequency dependence of mechanical loss tangent of PCCs $(\mathrm{Z}=\underline{1})$ with a change in the concentration of the complexed copolymer: (1) 0.1 and (2) $1.0 \mathrm{~g} / \mathrm{dl}$ 